\title{
Numerical Analysis of Ultimate Mechanical Characteristics of Suspension Bridges's Cable in High Temperature Fire
}

\author{
Jiarun Song, Xuehong Li, Weiqing Liu \\ Civil Engineering Department, Nanjing Tech University, Nanjing, China \\ Email address: \\ spencersong1996@163.com (Jiarun Song), lixuehongnj@163.com (Xuehong Li)
}

\section{To cite this article:}

Jiarun Song, Xuehong Li, Weiqing Liu. Numerical Analysis of Ultimate Mechanical Characteristics of Suspension Bridges's Cable in High Temperature Fire. Science Discovery. Vol. 9, No. 3, 2021, pp. 121-127. doi: 10.11648/j.sd.20210903.17

Received: April 19, 2021; Accepted: May 17, 2021; Published: May 24, 2021

\begin{abstract}
Relying on the joint research project, research is carried out on the ultimate mechanical characteristics of suspension bridge slings under high temperature fire. The establishment of a local transient temperature field heat transfer model based on the FDS software, and the analysis of the temperature distribution law of the fire source at different wind speeds. The most unfavorable fire condition is the combination of the fire source heat release rate of $300 \mathrm{MW}$ and the wind speed of $14 \mathrm{~m} / \mathrm{s}$; the overall structure model of the bridge is modeled by ANSYS software. Firstly, the modal analysis of the whole bridge model is carried out to verify the accuracy of the modeling, and then the thermal structural coupling analysis is carried out under the most unfavorable fire conditions to obtain the structural response of the whole bridge structure under the sling fire. According to the change trend of the structure response of the cable under fire, the fire resistance limit of the bridge structure is obtained as $460^{\circ} \mathrm{C}$; Under the most unfavorable fire burning situation, when the limit number of broken cable in the span is 3 , the cable will be caused into collapsing continuously; the composite protective layer has a significant effect on the fire resistance of the cable.
\end{abstract}

Keywords: Suspension Bridge Fire, Wind Speed Temperature Field, Cable Broke Continuously at High Temperature

\section{悬索桥高温火灾吊索极限力学特性数值分析}

宋佳润, 李雪红, 刘伟庆

南京工业大学土木工程学院, 南京, 中国

邮箱

spencersong1996@163.com (宋佳润), lixuehongnj@163.com(李雪红)

摘要: 以联合研究项目为依托, 针对悬索桥高温火灾吊索极限力学特性开展研究。建立基于FDS软件的局部瞬态温度 场传热模型, 分析不同风速火灾源的温度分布规律, 得出火灾源热释放率 $300 \mathrm{MW}$ 、风速为 $14 \mathrm{~m} / \mathrm{s}$ 的组合为最不利火灾 工况; 通过ANSYS软件对桥梁整体结构模型进行建模, 首先对整桥模型进行模态分析以验证建模的准确性, 接着在最 不利火灾工况下开展热-结构耦合分析, 得出整桥结构中吊索火灾下的结构响应。根据吊索火灾下的结构响应变化趋势, 得到吊索的耐火极限为 $460^{\circ} \mathrm{C}$; 最不利火灾烧断情况为跨中吊索极限断裂根数为 3 根时, 会引起吊索连续断裂。

关键词: 悬索桥火灾, 风速温度场, 吊索高温连续断裂 


\section{1. 引言}

火灾是经常发生、破坏性极大的灾害之一，严重威胁 公众安全以及社会发展。作为桥梁极端荷载之一的火灾燃 烧和爆燃对大桥造成的破坏不可估量, 高强度火源燃烧和 燃爆可能导致大桥局部构件破坏甚至整桥坍塌。因此, 开 展火灾状态下桥梁特别是大跨度的吊索桥抗火极限承载 能力研究，对保障人民的生命财产安全、预防火灾垮塌以 及灾后评估等具有显著的社会和经济效益, 现实意义重大。 桥梁结构抗火抗爆已引起交通管理部门及学者的高度关 注 [1-2]。引起桥梁使用过程中火灾事故的主要因素, 如运 输车辆的自燃以及大型油罐车的燃烧或集中爆燃等等, 各 国相关研究主要集中于桥梁结构材料的高温性能、桥梁火 灾数值模拟及火灾试验校核、火灾后桥梁承载能力计算简 化模型、桥梁防火防爆措施研究等。

大跨度的悬索桥主缆和吊索是主要承力结构体, 作 为多次超静定结构的索桥的吊索多采用平行钢束以及 钢丝绳或钢绞线作为承重材料, 受力复杂[3], 钢结构材 料的耐火性能差是一个致命弱点。结构材料的抗火性能 实验和数值模拟是目前国内外进行火灾研究的主要方 法。根据实验结果开展计算机数值模拟仿真, 依据火灾 场景参数进行大数据量模拟, 节约研究和测试的周期和 费用, 是火灾研究的重要手段 [4]。田伟 [5]对武汉市财鸟 鹉洲长江大桥汽车燃烧下的高温力学性能进行了研究。 袁旭东[6]对船舶油气爆燃火灾加强机制开展研究, 采用 CFD软件高质量网格分析了燃烧的细节以及标的物高 温力学性能。刘世忠等[7]以火灾下受损的大嶝大桥为背 景, 采用火灾结构分析软件FDS建立该桥热分析模型, 计算受损桥跨的温度场, 获得了桥梁高温温度场、损伤 范围与深度。陈玲珠等 [8]对比了中国规范、欧洲规范和 英国规范有关组合梁抗火设计, 并预测了组合梁的临界 温度和耐火极限。对火灾作用下桥梁的温度场数值模拟 基本采用简化方法, 将结构离散为线单元, 计算误差较 大[9-10]。

计算流体力学 (Computer Fluid Dynamics, 简称 CFD) 仿真软件是结合计算机和数值方法来求解流体力学的有 效工具, 能够获得火灾过程的细节数据。火灾燃烧过程 是一种湍流过程, 是不规则的非稳态流动现象, 可采用 大浴模拟方法(Large Eddy Simulation, LES)。有限元分 析软件ANSYS在结构力学动态响应特征分析中被广泛 采用。

本文以润扬大桥南汊桥结构特征为基础, 分析火灾 场景下吊索极限力学特性。利用有限元软件ANSYS, 建 立南汊桥全桥简化梁单元、关键部位实体单元的三维空 间热-结构耦合有限元模型, 在大幅减少模型单元数目的 基础上, 既精确模吊索的三维空间温度场分布, 又能将 吊索同主缆和悬索桥全桥总体进行连接, 计算吊索高温 下的极限力学性能。采用CFD软件进行火灾燃烧环境场 和吊索力学特征仿真计算。结合两种软件的优势, 针对 极限燃烧, 进行吊索高温火灾力学特性仿真分析和复合 层材料防护吊索高温特性分析。

\section{2. 悬索桥热-力-结构耦合建模}

\section{1. 标的工程概况}

润扬大桥是中国第一大跨径的组合型桥梁, 是技术最 复杂含量最高的现代化特大型桥梁工程, 是一座刚柔相济 的组合型桥梁, 大桥全长为 35.66 公里, 桥面平均宽 31.5 米(行车道宽30米), 全线采用双向六车道高速公路标准设 计, 由北接线、北汊桥、世业洲互通高架桥、南汊桥 (如 图1所示) 延伸段等部分组成; 主桥包括北汉桥、世业洲 互通高架桥和南汉桥, 长7.21公里; 南汊桥主桥为钢箱梁 悬索桥, 索塔高 $209.9 \mathrm{~m}$, 两根主缆直径为 $0.868 \mathrm{~m}$, 长度 2600 米, 跨径布置是 $470 \mathrm{~m}+1490 \mathrm{~m}+470 \mathrm{~m}$; 北汉桥为主双塔双 索面钢箱梁斜拉桥, 跨径布置是 $175.4 \mathrm{~m}+406 \mathrm{~m}+175.4 \mathrm{~m}$, 倒 $\mathrm{Y}$ 型索塔高 $146.9 \mathrm{~m}$; 索桥主塔高227.21米, 大桥钢箱梁 总重34000吨。

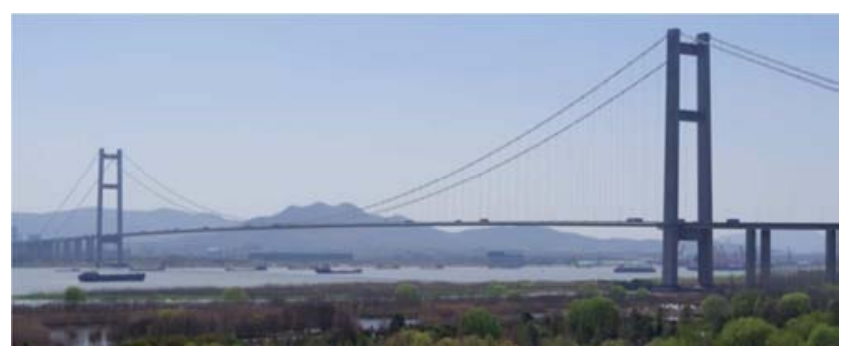

图1 润扬大桥南汊桥侧视图。

\section{2. 悬索桥热-力-结构耦合建模理论基础与方法}

\subsection{1. 耦合建模理论基础}

基于南汊桥结构特征分析火灾场景下吊索极限力学 特性仿真分析, 所用的结构力学模型和流体力学模型表征 如下:

（1）ANSYS热分析方程

根据燃烧分析，设定在时间段对结构应用能量能守恒 进行求解 [11-12], 得到火灾下结构三维瞬态热传导方程:

$$
c \rho \frac{\partial u}{\partial t}=\frac{\partial}{\partial x}\left(k \frac{\partial u}{\partial x}\right)+\frac{\partial}{\partial y}\left(k \frac{\partial u}{\partial y}\right)+\frac{\partial}{\partial z}\left(k \frac{\partial u}{\partial z}\right)
$$

式中: $u$ 为物体在位置 $(x, y, z)$ 处、时刻 $t$ 时的温度; $\mathrm{t}$ 为受火时间; $\rho$ 为材料的质量密度; $\mathrm{c}$ 为材料的比热容; $x$, $y$ 和 $z$ 为结构的三维坐标; $k$ 为材料的导热系数。桥梁遭受 火灾时受火面为第 3 类边界条件, 即

$$
\left.\left(\frac{\partial \theta}{\partial n}+h \theta\right)\right|_{\Gamma}=\Psi(x, y, z)
$$

式中: $\mathrm{h}$ 为已知正数; $\Psi$ 为结构 $\Omega$ 的边界曲面 $\Gamma$ 上温度 的已知函数; $\theta$ 为温度; $\partial \theta / \partial \mathrm{n}$ 为温度梯度。确定结构的 瞬态温度场后, 将温度作为热荷载进行结构分析, 温度应 力等效成由温度变化引起的初应变。根据应力与位移平衡 方程及最小势能原理可得 


$$
\begin{array}{r}
\frac{\partial \pi}{\partial \delta^{e}}=\left(\iiint_{\Omega} \mathbf{B}^{\mathrm{T}} \mathbf{D} \mathbf{B} d \Omega\right) \boldsymbol{\delta}^{e}-\iiint_{\Omega} \mathbf{B}^{\mathrm{T}} \mathbf{D} \varepsilon_{0} d \Omega \\
-\iiint_{\Omega} \mathbf{N}^{\mathrm{T}} p_{v} d \Omega-\iiint_{B_{2}} N^{\mathrm{T}} p_{s} d B=0
\end{array}
$$

式中: $\delta^{\mathrm{e}}$ 为位移向量; $B$ 和 $S$ 为应变矩阵和应力矩阵; $N$ 为形函数矩阵; $D$ 为弹性矩阵; $P_{\mathrm{s}}$ 为分布面力; $P_{\mathrm{v}}$ 为体积 力; $\pi$ 为结构的势能。解出结点位移 $\delta$, 利用单元特性计算 得到单元应力。

(2) 大浴模拟方程

流体力学仿真所采用的大涡模拟方法的基本思想是 对湍流中大于网格尺度的浴利用 $\mathrm{N}-\mathrm{S}$ 方程直接进行数值计 算, 而对比网格尺度小的浴则通过建立模型的方法进行模 拟。这种方法结合了直接数值模拟和雷诺平均方法的优点, 同时规避了两者的缺点。

大浴模拟通过滤波将流动变量 $\mathrm{f}(\mathrm{x}, \mathrm{t})$ 分解成大尺度量 $\bar{f}(x, t)$ 和小尺度量 $f^{\prime}(x, t)$, 其中大尺度量为可解量, 可直接在主网格中求解, 而小尺度的量则需通过相应的亚 网格模型进行封闭。

$$
f(x, t)=\bar{f}(x, t)+f^{\prime}(x, t)
$$

其中, $\bar{f}(x)=\int_{v} f\left(x^{\prime}\right) F\left(x-x^{\prime}\right) d x^{\prime}, \mathrm{F}(\mathrm{x})$ 为滤波函数。

常用的滤波函数有盒式滤波、傅式截断滤波、高斯滤 波以及Favre滤波等。

本文所使用的滤波函数为Favre滤波。这种滤波函数 用于可压缩流动问题时, N-S 方程较容易封闭, 因而受到 广泛应用。

$$
\bar{\rho} \tilde{f}(x)=\int \rho f^{\prime}(x) F\left(x-x^{\prime}\right) d x^{\prime}
$$

式中顶标“—”表示空间滤波，而顶标“ ”表示Favre滤 波。对可压缩N-S方程进行Favre滤波, 略去非线性项可得 连续性方程、动量方程、能量方程和状态方程如式（6）(9) 所示。

$$
\begin{gathered}
\frac{\partial \bar{\rho}}{\partial t}+\frac{\partial}{\partial x_{j}}\left(\bar{\rho} \tilde{u}_{j}\right)=0 \\
\frac{\partial \bar{\rho} \tilde{u}_{i}}{\partial t}+\frac{\partial}{\partial x_{j}}\left(\bar{\rho} \tilde{u}_{i} \tilde{u}_{j}\right)=-\frac{\partial \bar{p}}{\partial x_{i}}+\frac{\partial \sigma_{i j}}{\partial x_{j}} \\
\frac{\partial \bar{\rho} \tilde{E}}{\partial t}+\frac{\partial}{\partial x_{j}}\left(\bar{\rho} \tilde{u}_{j} \tilde{E}+\bar{p}_{j}\right)=-\frac{\partial \bar{q}_{j}}{\partial x_{j}}+\frac{\partial}{\partial x_{j}}\left(\tilde{u}_{i} \sigma_{i j}\right) \\
\bar{p}=\bar{\rho}\left(c_{p}-c_{v}\right) T
\end{gathered}
$$

其中, 变量 $\rho, u_{i}, p$ 和 $E$ 分别为密度、速度、压力和 比总能, $\sigma_{\mathrm{ij}}$ 为有效粘性应力张量, $\mathrm{q}_{\mathrm{j}}$ 为有效热通量, $\mathrm{c}_{\mathrm{p}}$ 为
定压比热容, $c_{\mathrm{v}}$ 为定容比热容。本文采用可压缩湍流的 Smagorinsky亚网格模型。

\subsection{2. 南汉桥悬索桥热-力-结构耦合建模}

润扬悬索桥钢箱梁采用BEAM189单元，根据CAD图 纸自定义截面, 实现整桥梁单元建模。混凝土桥塔采用 solid45单元, 分节段建立多个截面, 完成桥塔建模。主缆 和吊索采用link10建模, 打开ANSYS大变形考虑索的应力 刚化效应, 通过设置初始应变与初始预应力实现主缆和吊 索的预拉力。吊索和主梁、主缆和桥塔之间约束采用主从 约束, 主梁与桥塔的约束根据实际支座刚度设置 combine14弹簧单元, 主缆接地端约束全部自由度。

建立润扬悬索桥ANSYS模型, 如图2, 图3所示。进 行模态分析, 对比润扬悬索桥实测模态数据, 验证模型建 立的可靠性，模态数据对比如表1所示。

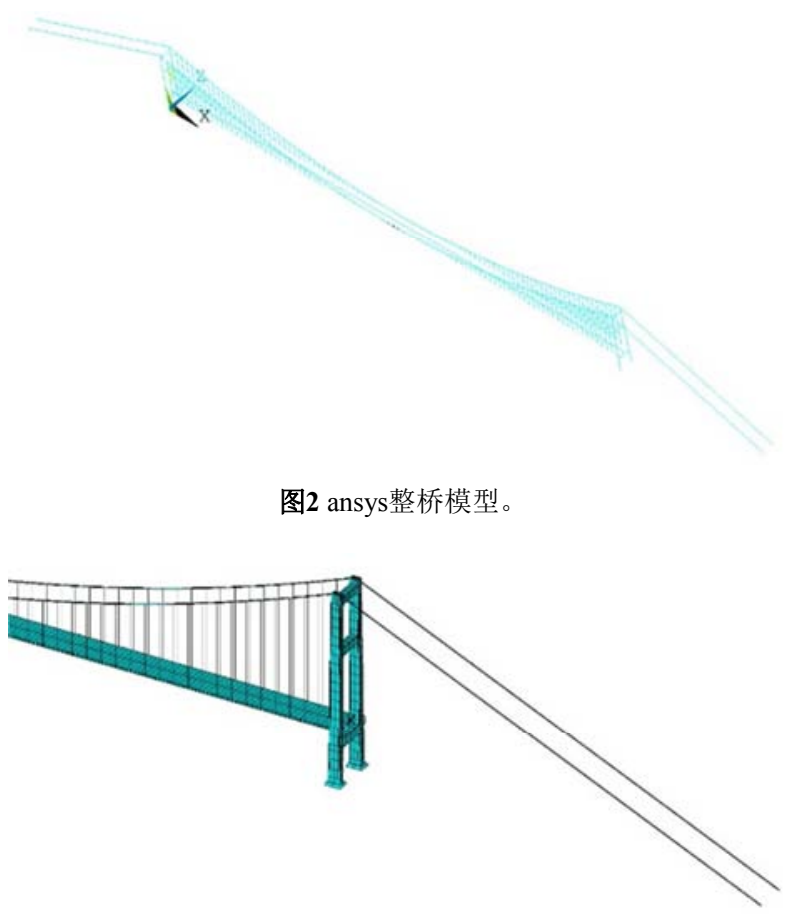

图3 局部细节模型。

\subsection{3. 热-力-结构耦合建模有效性验证}

为验证本文中热-力-结构耦合建模方法的有效性, 参 考文献[13]研究选取1989年英国钢结构技术实验室的抗火 试验进行模型验证。验证结果显示: 温度和挠度的试验值 和有限元计算结果非常接近, 证明ANSYS热一结构耦合建 模有效。火灾燃烧阶段钢梁各部位的温度试验值与有限元 计算值的对比结果如图4所示, 试验梁跨中挠度试验值与 有限元计算值的对比结果如图5所示。

表1 润扬悬索桥ANSYS模态分析。

\begin{tabular}{llll}
\hline \multirow{2}{*}{ 阶态数 } & 自振频率 & & \\
\cline { 2 - 4 } & 有限元计算频率 & 设计院设计模态 & 误差 \\
\hline 1 & 0.0536 & 0.0496 & $8.06 \%$ \\
2 & 0.0967 & 0.0895 & $8.04 \%$ \\
3 & 0.1209 & 0.1250 & $3.3 \%$ \\
\hline
\end{tabular}




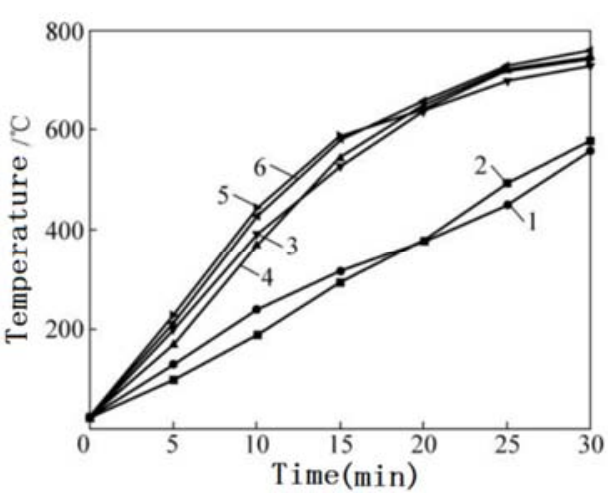

图4 钢梁各部位温度随时间的变化。

1-顶板温度有限元值 2-顶板温度试验值 3-底板温度有限元值 4-底 板温度试验值 5 -腹板温度有限元值 6 - 腹板温度试验值

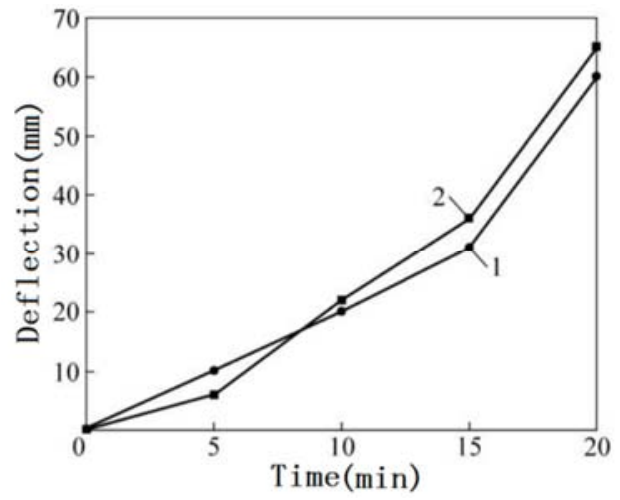

1-跨中挠度有限元值 2-跨中挠度试验值

图5 试验梁跨中挠度随时间的变化。

\section{3. 火源燃烧风场影响分析}

道桥建设的桥梁主体基本都高于所属环境，外环境的 风场易于形成, 风力的强弱对火源燃烧有重要影响。因此 必须考虑风速的大小对燃烧状态, 特别是对燃烧温度分布 的影响。

对于燃烧过程关键点检测火焰温度, 风速的大小对于

测点温度影响的检测结果如图6, 图7, 图8所示, 即 不同的风速下被测点温度随风速发生变化: 风速在 0 和 $2 \mathrm{~m} / \mathrm{s}$ 时的最大温度都超过 $1000^{\circ} \mathrm{C}$, 当风速为 $6 \mathrm{~m} / \mathrm{s}$ 时的最大 温度为 $625^{\circ} \mathrm{C}$, 风速为 $10 \mathrm{~m} / \mathrm{s}$ 时的最大温度为 $565^{\circ} \mathrm{C}$, 风速 为 $14 \mathrm{~m} / \mathrm{s}$ 时的最大温度为 $520^{\circ} \mathrm{C}$, 风速达到 $6 \mathrm{~m} / \mathrm{s} 、 10 \mathrm{~m} / \mathrm{s}$ 、 $14 \mathrm{~m} / \mathrm{s}$ 时火焰的温度降低了, 不同测点结论一致。
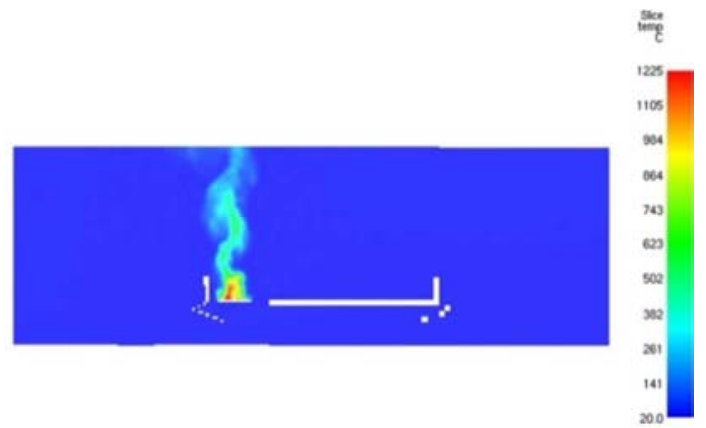

图6 风速 $0 \mathrm{~m} / \mathrm{s}$ 下火源燃烧云图。

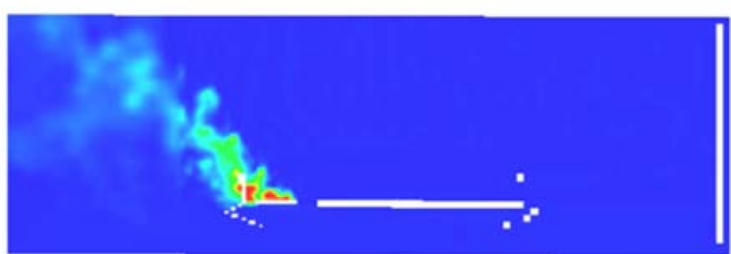

图7 风速 $6 \mathrm{~m} / \mathrm{s}$ 下火源燃烧云图。

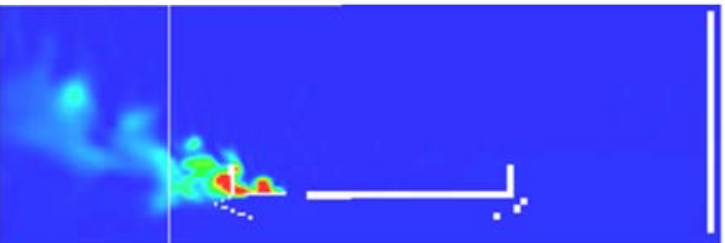

图8 风速 $10 \mathrm{~m} / \mathrm{s}$ 下火源燃烧云图。

风速改变使空气流速的变化, 也是火焰温度随之变化 而变化, 但火焰温度的变化并不是单调的。也就是说风速 从微小增大时会增加火焰温度, 而当风速过高时并不会使 火焰温度增加, 反而会使火焰温度降低。随着风速的增加 火焰温度下降, 是由于风速的增加使冷空气的介入增加, 热空气与冷空气的交换速度也加快, 温度场扩散速度加快, 从而使火焰的温度被拉低。

充足氧气使火焰燃烧的更剧烈, 介质热量释放的更完 全, 够促进火焰与空气的热交换, 使热蒸发的气体燃料扩 散的更快燃烧释放的热量也更多。

因此本文针对吊索极限燃烧，温度场仿真分析风速的 大小选取为 $14 \mathrm{~m} / \mathrm{s}$ 。吊索温度场如图9所示。

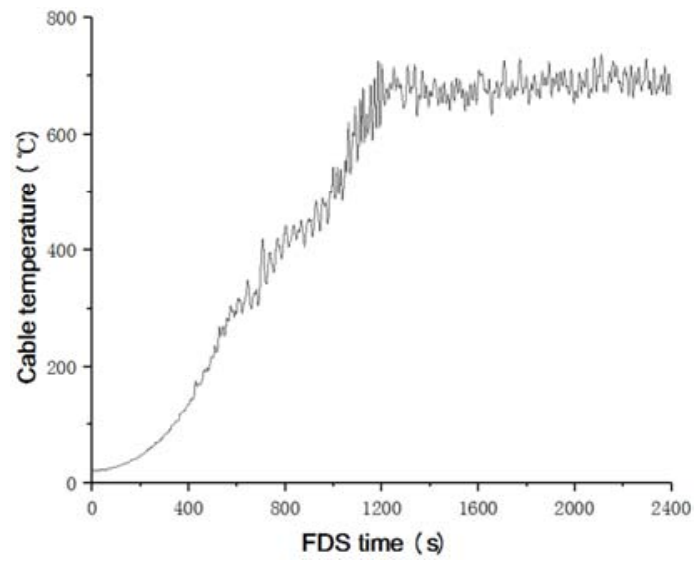

图9 吊索温度场。 


\section{4. 吊索材料特性参数和火焰燃烧升温历程确定}

\subsection{1. 吊索钢丝热工参数选择}

润扬大桥南汊桥吊索材料为镀锌高强钢丝, 其热工参 数选取在国内外学者对钢丝热工参数大量研究的基础上 参照文献[14]中的研究成果, 对钢丝的热工参数进行取值 如式 (12)所示。

$$
\begin{aligned}
& \lambda(\theta)= \begin{cases}-0.022 \theta+48 & 0 \leqslant \theta \leqslant 900^{\circ} \mathrm{C} \\
28.2 & \theta>900^{\circ} \mathrm{C}\end{cases} \\
& C(\theta)=38.1 \times 10^{-8} \theta^{2}+20.1 \times 10^{-5} \theta+0.473 \\
& \alpha(\theta)= \begin{cases}(0.004 \theta+12) \times 10^{-6} & \theta<1000^{\circ} \mathrm{C} \\
16 \times 10^{-6} & \theta>1000^{\circ} \mathrm{C}\end{cases} \\
& E(\theta)=\frac{E}{1.03+32 \times(\theta+108)^{6} \times 10^{-18}} \\
& f(\theta)=\left[0.99+4.75 \times 10^{-4} \times \theta-5.57 \times 10^{-6} \times \theta^{2}+\right. \\
& \left.1.02 \times 10^{-9} \times \theta^{3}+4.55 \times 10^{-12} \times \theta^{4}\right] f
\end{aligned}
$$

式中: $\lambda(\theta)$ 为钢的导热系数函数; $C(\theta)$ 为钢的比热函 数; $\alpha(\theta)$ 为钢的热膨胀系数函数; $\mathrm{E}(\theta)$ 为钢的弹性模量函 数; $E$ 为常温下钢的弹性模量; $f(\theta)$ 为钢的强度函数; $f$ 为 常温下钢的强度。钢的密度和泊松比随温度变化很小, 取 常温值 $7.85 \mathrm{t} / \mathrm{m}^{3}$ 和 0.3 。

\subsection{2. 火焰燃烧升温历程确定}

润扬大桥是中国第一大跨径的组合型桥梁，是技术最 复杂含量最高的现代化特大型桥梁工程, 是一座刚柔相济 的组大桥, 桥上通行车辆繁忙且数量大品种多。不同燃烧 车辆的温升过程和达到的最高温度也不同。田伟定义的小 型车辆如小汽车、客车和货车等燃烧曲线 [15]和本文研究 采用的法国 $\mathrm{HC}_{\mathrm{inc}}$ 升温曲线如图 10 所示。 $\mathrm{HC}$ (碳氢燃烧 HYDROCARBON）升温曲线最初主要用于研究海洋工程 上燃烧分析, 后来逐步应用分析由汽车油罐车上的油类物 质燃烧所造成的火灾特性。油罐车火灾时爆发力特别大, $10 \mathrm{~min}$ 内火源区域温度可达到 $1000^{\circ} \mathrm{C}$ 以上, 而 $\mathrm{HC}_{\text {inc }}$ 温升曲 线是 $\mathrm{HC}$ 曲线的强化版，是在 $\mathrm{HC}$ 曲线基础上适合的乘以放 大倍数。因此, 对桥梁吊索极限火灾受火区域采用HCinc 曲线是适合的。本文油罐车燃烧升温方程如式 (13) 所示。

$$
T=20+1080\left(1-0.325 e^{-0.167 t}-0.675 e^{-2.5 t}\right)
$$

式中: $\mathrm{T}$-t时刻的温度 $/{ }^{\circ} \mathrm{C}, \mathrm{t}$-时间 $/ \mathrm{min}$ 。

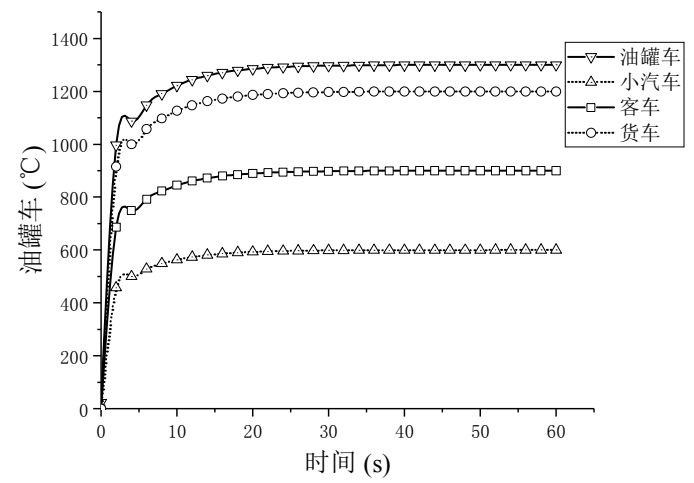

图10 四种车型升温曲线对比图。

\section{3. 索桥高温火灾吊索极限力学特性数值分析}

\section{1. 火源极限燃烧吊索失效状态分析}

润扬大桥南汊吊索由 109 根直径 $5 \mathrm{~mm}$ 的高强平行钢丝 束组成, 外层包覆 $6 \mathrm{~mm}$ 聚乙烯保护套。高强钢丝及钢丝绳。 吊索钢丝常温下弹性模量 $2.0 \times 10^{5} \mathrm{MPa}$, 密度 $7850 \mathrm{~kg} / \mathrm{m}^{3}$, 泊松比 0.1 。吊索钢丝常温下强度级别为 $1670 \mathrm{MPa}$ 。其结构 如图11所示。

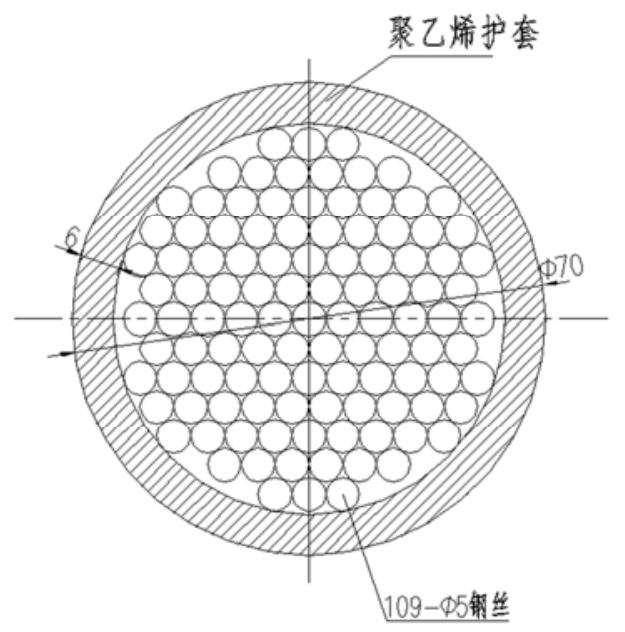

图11 吊索断面图。

$300 \mathrm{MW}$ 油罐车燃烧, 风速为 $14 \mathrm{~m} / \mathrm{s}$ 火灾工况下, 温度 上升趋势如图所示。当温度达到 $460^{\circ} \mathrm{C}$ 左右时, 吊索应力 大于此时吊索抗拉强度。如图12所示, 所以吊索的耐火极 限温度为 $460^{\circ} \mathrm{C}$ 。

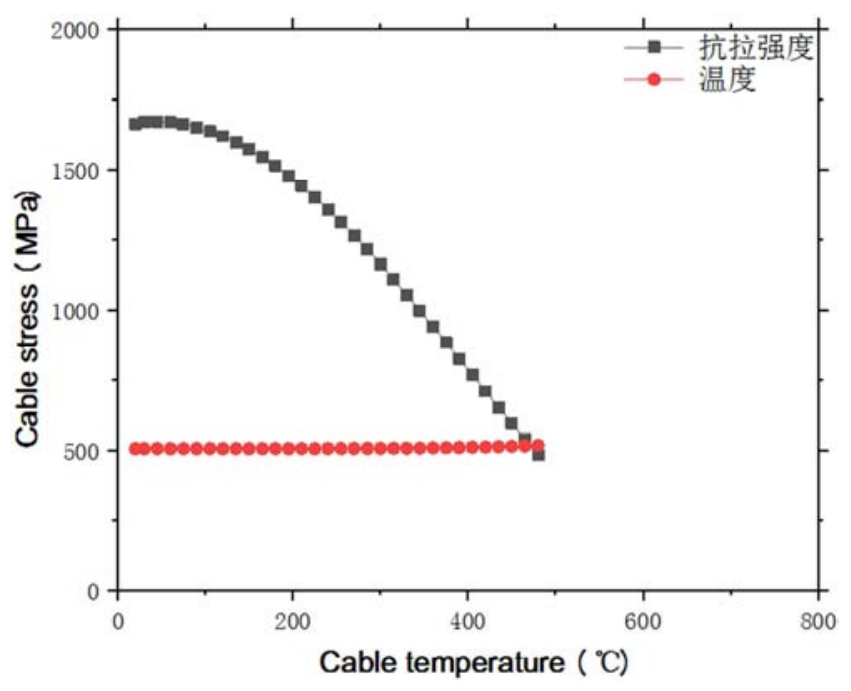

图12 吊索随温度变化的应力图。

\section{2. 高温燃烧吊索连续断裂力学特性仿真}

吊索大桥的每根吊索设计承力依据悬链线特征作等 拉力或等应力分配。因此, 如果某个吊索或多个吊索受火 灾发生断裂, 将可能发生雪崩式的连续断裂现象, 将对整 个桥梁结构安全带来的危害将是致命的。 
下表中L-M指的是中间吊索（link-middle），L-L1指 的是中间吊索的左边第一个吊索（link-lift 1)。由于本模 型是对称结构, 列出了一半吊索中内力值变化较明显的数 据, 如表2所示:

表2 单根(跨中) 吊索断裂剩余吊索应力值。

\begin{tabular}{llll}
\hline \multirow{2}{*}{ 吊索编号 } & \multicolumn{3}{l}{ 不同状态下吊索应力 } \\
\cline { 2 - 4 } & 成桥状态 & 高温断裂状态 & 直接断裂状态 \\
\hline L-R1 & 511.2 & 801.9 & 823.9 \\
L-M & 510.4 & 无 & 无 \\
L-L1 & 512.8 & 802.1 & 824.4 \\
L-L2 & 511.9 & 537.0 & 541.2 \\
L-L3 & 501.4 & 522.4 & 525.6 \\
L-L5 & 501.2 & 523.6 & 526.6 \\
L-L6 & 501.3 & 524.1 & 527.1 \\
L-L7 & 501.4 & 524.4 & 527.3 \\
L-L8 & 501.5 & 524.5 & 527.5 \\
L-L9 & 501.5 & 524.6 & 527.5 \\
L-L10 & 501.6 & 524.7 & 527.6 \\
L-L11 & 501.6 & 524.7 & 527.6 \\
L-L12 & 501.7 & 524.8 & 527.7 \\
L-L13 & 501.8 & 524.8 & 527.8 \\
L-L14 & 501.8 & 524.9 & 527.9 \\
L-L15 & 501.9 & 525.0 & 528.0 \\
\hline
\end{tabular}

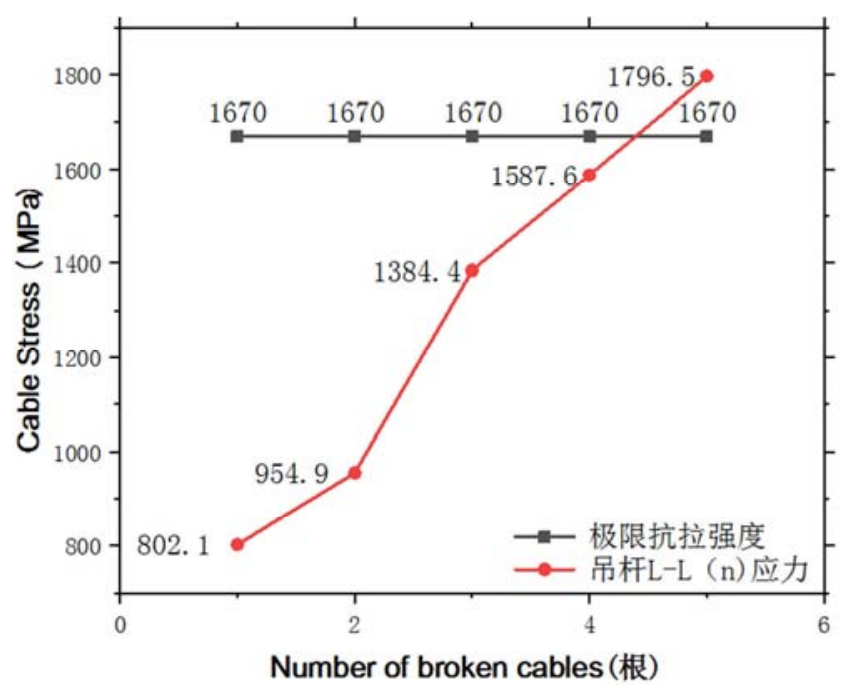

图13 吊索L-L(n)应力。

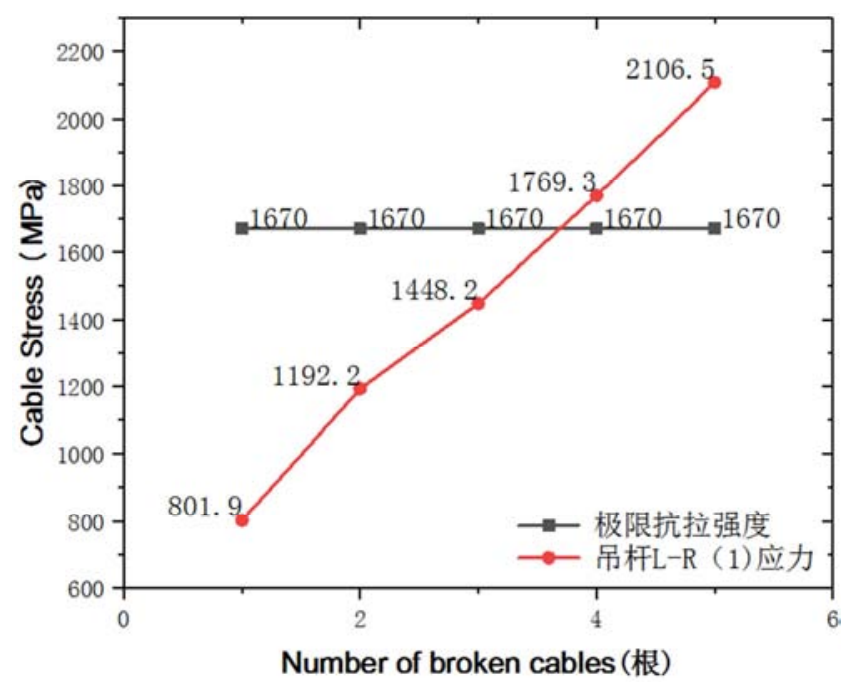

图14 吊索L-R(1)应力。
从图13, 图14所示, 高温断裂吊索数量达到三根时, $\mathrm{L}-\mathrm{L}$ (3) 应力为 $1384.4 \mathrm{Mpa}, \mathrm{L}-\mathrm{R}(1)$ 应力为 $1448.2 \mathrm{Mpa}$, 已经 较为接近极限抗拉强度 $1670 \mathrm{Mpa}$; 当断裂根数达到四根时, $\mathrm{L}-\mathrm{R}(1)$ 应力为 $1769.3 \mathrm{Mpa}$, 超过极限抗拉强度, 所以跨中 吊索极限断裂根数为 3 根时, 会引起吊索连续倒塌。

\section{4. 结论}

（1）风速与火源的燃烧热释放功率存在强相关关系, 随着风速的增加火源热释放功率由弱增强, 风速到达一定 数值时, 火源热释放功率达到最大, 这是由于风供氧助燃 的同时流动带走热量, 到达温升和扩散的动态平衡, 分析 认为风速 $14 \mathrm{~m} / \mathrm{s}$, 火灾源热释放率 $300 \mathrm{MW}$ 为最不利吊索火 灾工况;

（2）跨中吊索断裂会引起相邻吊索连续断裂，分析 认为, 当吊索极限断裂根数为 3 根时, 会引起吊索连续断 裂, 造成大桥失去功能或坉塌。

（3）在满足索桥设计承重载荷安全系数的前提下, 有效载荷之一的环境温度载荷是对吊索的力学性能影响 显著, 随着火源对吊索的加温, 吊索抗拉强度随之下降, 通过随温度变化的吊索极限抗拉强度与吊索应力对比, 吊 索温度耐火极限为 $460^{\circ} \mathrm{C}$ 。

\section{致谢}

本文为江苏省前瞻性联合研究项目《城市桥梁群智能 管理关键技术研究及系统开发》(BY2016005-12)的阶段性 成果之一。

\section{参考文献}

[1] 张岗, 贺栓海, 宋超杰, 黄侨, KODUR V K, 张永飞.钢结 构桥梁抗火研究综述 [J].中国公路学报, 2021, 34(01):1-11.

[2] 李艳, 汪剑, 周国华.大跨径悬索桥缆索体系抗火设计研究 [J].公路, 2018，63(05):94-101.

[3] 杨光武, 徐宏光, 张强.马鞍山长江大桥三塔悬索桥关键技 术研究[J].桥梁建设, 2010(5):7-11。

[4] 丛北华, 廖光煌, 韦亚星.计算机模拟在火灾科学与工程研 究中的应用[J].防灾减灾工程学报, 2003, 23(2):63-69。

[5] 田伟.武汉鹦鹉洲长江大桥汽车燃烧下高温力学性能与风 险防范措施研究 [D].武汉: 武汉理工大学土木工程与建筑学 院, 2014:87-98。

[6] 袁旭东, 船舶油气爆燃加强机制研究[D].哈尔滨工业大学, 2017:8-63。

[7] 刘世忠, 马朝旭, 李丽园, 刘欣益等.火灾下PC箱梁的损伤 评估与加固设计 [J].桥梁建设, 2014, 44(6):94-100。

[8] 陈玲珠, 蒋首超, 李国强.不同规范组合梁抗火设计方法的 比较和分析[J].防灾减灾工程学报, 2015, 35(1):69-78。 
[9] KODUR V, AZIZ E, DWAIKAT M.Evaluating fire resistance of steel girders in bridges[J].Journal of Bridge Engineering, 2013, 18(7):633-643。

[10] 熊伟, 李耀庄, 严加宝. 火灾作用下钢筋混凝土梁温度场数 值模拟及试验验证 [J].中南大学学报(自然科学版), 2012, 43(7):2839-2843。

[11] LIU Fentao, WU Bo, WEI Demin.Failure modes of reinforced concrete beams strengthened with carbon fiber sheet in fire $[\mathrm{J}]$. Fire Safety Journal，2009，44(7):941-950。
[12] 吕学涛, 杨华, 张素梅.三面受火的方钢管混凝土柱耐火极 限[J].自然灾害学报，2012，21(3):198-203。

[13] 王莹等.大跨经悬索桥缆索抗火模拟方法 [J].中南大学学报 (自然科学版), 2012, 47(6)2091-2099。

[14] 姬芬芬.FPSO 的火灾风险评估 [D]. 宁波: 宁波大学, 2017:26-35。

[15] 田伟.武汉鹦武洲长江大桥汽车燃烧下高温力学性能与风 险防范措施研究[D].武汉:武汉理工大学, 2014:61-62。 